\title{
Towards Wide-Bandwidth Ultra-Flat FOPAs
}

\author{
Vladimir Gordienko ${ }^{1}$, Marc Stephens ${ }^{1}$, and Nick Doran ${ }^{1}$ \\ ${ }^{1}$ Aston Institute of Photonic Technologies, Aston University, B4 7ET, Birmingham, UK \\ Tel: (44) 121204 3512,e-mail: gordienv@aston.ac.uk
}

\begin{abstract}
Fibre optical parametric amplifiers (FOPAs) offer the potential for high gain and $>100 \mathrm{~nm}$ bandwidth at arbitrary wavelengths for increased transmission capacity. We will cover the main principles of the FOPA and discuss our approach to obtaining broad flat gain and performance improvement via simultaneous Raman amplification.

Keywords: optical parametric amplifiers, broadband amplifiers, Raman scattering.
\end{abstract}

\section{INTRODUCTION}

Modern optical transmission systems are approaching the nonlinear Shannon limit, and techniques are being explored to extend performance beyond it [1]. An alternative way of enhancing capacity is to increase the transmission bandwidth (BW). Some fibres, such as OFS AllWave ${ }^{\circledR}$ allow low-loss $(<0.32 \mathrm{~dB} / \mathrm{km})$ transmission over a wide range $(1310-1625 \mathrm{~nm})$. This presents an available BW of $44 \mathrm{THz}$, with only $\sim 9 \mathrm{THz}$ covered by $\mathrm{C}$ and L-band Erbium-doped fibre amplifiers (EDFAs). The development of amplifiers capable of providing one-stage amplification over the full transmission band would offer a fivefold transmission capacity increase.

The most promising amplifiers capable of operating in that window are the fibre Raman amplifier (FRA) and the FOPA. However, the gain BW of FRA has so far been limited to $\sim 100 \mathrm{~nm}$, and requires multiple $(>5)$ pumps [2]. Conversely, a single-pump FOPA with a total gain $\mathrm{BW}>30 \mathrm{THz}$ has been demonstrated [3]. Additionally, the FOPA has a higher gain coefficient than FRA, and the FOPA can operate in a phase-sensitive regime enabling noise figures below $3 \mathrm{~dB}$ [4]. However, the FOPA still has numerous challenges - for example, the unidirectional nature of the gain prohibits backward pumping and means pump noise can transfer to signals. Secondly, the FOPA suffers from four-wave mixing between signals leading to crosstalk. Thirdly, pump power is limited by stimulated Brillouin scattering (SBS), and techniques used to mitigate it (such as pump dithering) cause signal degradation [5]. Finally, it is difficult to obtain polarization insensitive gain, although recent progress has been made [6].

This paper reviews the FOPA principle and recent achievements of wide gain BW operation suitable for signal amplification. We will explore how Raman gain from the parametric pump can be used to extend FOPA gain BW.

\section{BROADBAND FOPA}

The single-pump FOPA gain coefficient depends on pump power $P$, fibre nonlinearity $\gamma$ and propagation constant mismatch between pump, signal and idler $\Delta \beta$ as shown in (1) [7]. The nonlinear medium is typically a Highly Nonlinear Fibre (HNLF). Considering co-polarized waves, the maximum gain coefficient $g_{\max }=\gamma P$ is achieved when $\Delta \beta=-2 \gamma P$. This condition is termed 'phase-matched'. When $\Delta \beta$ is in a range from 0 to $-4 \gamma P$, but not equal to $-2 \gamma P$ parametric gain is strongly reduced. For $\Delta \beta$ outside of this range, the FOPA gain coefficient is imaginary.

$$
\begin{gathered}
g=\sqrt{-\Delta \beta \cdot(4 \gamma P+\Delta \beta)} / 2 \\
\Delta \beta=\beta_{s}+\beta_{i}-2 \beta_{p}=\beta^{(2)}\left(\Delta \omega_{s}\right)^{2}+\frac{\beta^{(4)}}{12}\left(\Delta \omega_{s}\right)^{4}
\end{gathered}
$$

Equation (2) defines $\Delta \beta$ for the single-pump FOPA, where $\beta_{s}, \beta_{i}$, and $\beta_{p}$ are propagation constants of signal, idler and pump waves respectively, and $\beta^{(2)}$ and $\beta^{(4)}$ are second- and fourth-order derivatives of $\beta_{p}$ (higher orders ignored), and $\Delta \omega_{s}$ is the angular frequency offset between the signal and the pump.

$$
G(z)=\left|\frac{\gamma P}{g} \sinh (g z)\right|^{2}+1
$$

The signal gain $G$ can be approximated by (3), where $z$ is the propagation distance. Figure 1 shows a typical single-pump FOPA gain spectrum calculated using (3). Unless $\beta^{(2)}$ is very small when pump is very close to zero-dispersion wavelength $\lambda_{0}$, the $\beta^{(2)}$-term dominates over the $\beta^{(4)}$-term in (2) over a wide range around the pump. In this case $\Delta \beta$ takes a parabolic shape and changes quickly across the range where gain coefficient is real. It results in a significant gain variation (GV) across the gain spectrum (Fig. 1). Near the pump $\Delta \beta$ is close to zero, so moderate quadratic gain is observed. As signal detuning from the pump increases, $\Delta \beta$ reaches a value of $-2 \gamma P$ and gain increases up to its maximum featuring an exponential gain growth. Further increase of signal 
detuning from the pump leads to phase mismatch and the gain correspondingly reducing to near zero. Note that gain magnitude is defined by nonlinear phase shift $\gamma P L$, where $L$ is HNLF length. Overall, even though the FOPA can provide high gain and wide BW, typically the GV across the spectrum is very high. Therefore, not only would high loss gain flattening filters be required for broadband operation, but significant penalties would be induced due to nonlinear crosstalk occurring between excessively amplified signal channels near the gain peak.

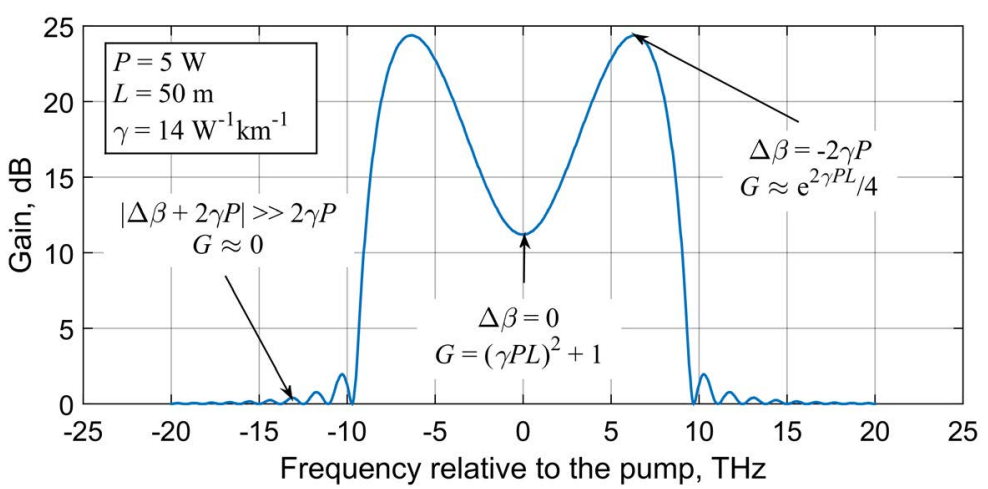

Figure 1. Example analytical FOPA gain spectrum from Eq. (3).

A flat and broad gain spectrum can best be achieved using short lengths of HNLF. This approach requires much higher pump power to obtain high gain, but has two advantages. First, wider gain BW can be obtained due to the higher pump power whilst keeping the peak gain the same. Secondly, short fibres suffer less from longitudinal dispersion fluctuations, giving more control over the gain spectrum shape when tuning pump wavelength. Thus, by positioning the pump very close to $\lambda_{0}$ at the anomalous dispersion side of HNLF possessing positive $\beta^{(4)}$ coefficient, the two terms in Eq. (2) can be cancelled out over a wide range. In this way $\Delta \beta \approx 0$ and gain is not phase-matched, but gain is almost constant over wide $\mathrm{BW}$.

\subsection{Experimental setup}

Figure 2 shows the setup for an experimental study of single-pump broadband FOPA gain spectra. A continuous pump wave generated from a $100 \mathrm{kHz}$ linewidth tuneable laser was phase modulated with radio frequency (RF) tones at $100 \mathrm{MHz}, 320 \mathrm{MHz}$ and $980 \mathrm{MHz}$ to mitigate SBS. The pump was amplified by a high-power EDFA and filtered using a circulator and a tuneable fibre Bragg grating (FBG) to remove EDFA noise from the pump, and to combine the pump with a signal probe. The pump and signal were guided into various lengths of dispersion stable HNLF [8]. The key datasheet parameters of the HNLF were: $\lambda_{0}=1550.5 \pm 1 \mathrm{~nm}$, $\gamma=14 \mathrm{~W}^{-1} \cdot \mathrm{km}^{-1}$, dispersion slope $S=0.044 \mathrm{ps} \cdot \mathrm{nm}^{-2} \cdot \mathrm{km}^{-1}$ and $\beta^{(4)} \sim 10^{-56} \mathrm{~s}^{4} \cdot \mathrm{m}^{-1}$, measured at $1550 \mathrm{~nm}$. To accurately measure the FOPA gain [9], a depolarized supercontinuum [10] was used as an input probe, ranging from $1520 \mathrm{~nm}$ to $1700 \mathrm{~nm}$. A calibrated set of tap couplers and power meters and an optical spectrum analyzer (OSA) were used to non-intrusively measure pump power and optical spectra at the input and output of the HNLF. The pump polarisation could be tuned using a polarisation controller placed at the input to the HNLF.

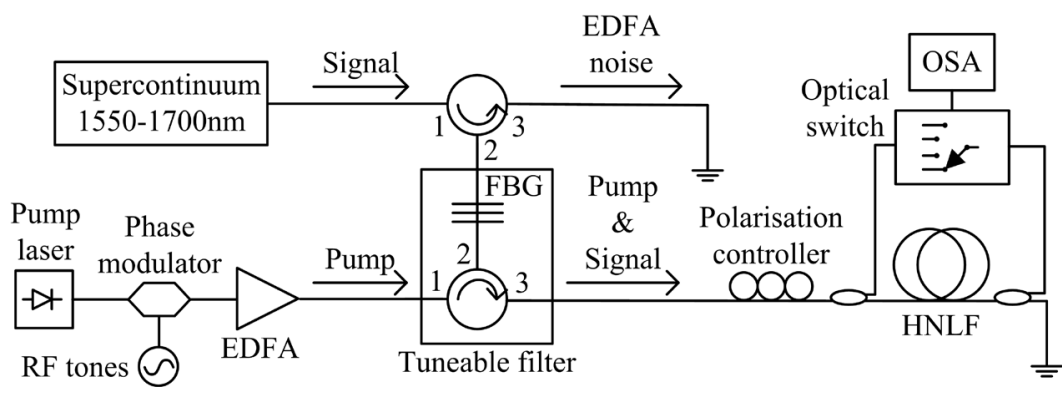

Figure 2. Experimental setup for FOPA gain spectrum analysis.

\subsection{Experimental results and discussion}

The blue curve in Fig. 3 shows a gain of $9.6 \pm 0.5 \mathrm{~dB}$ over $12.9 \mathrm{THz}$ obtained using the approach discussed above in a $25 \mathrm{~m}$ long fibre, and using a pump power of $9 \mathrm{~W}$ [11]. Gain achieved beyond $10.5 \mathrm{THz}$ is attributed mainly to Raman gain induced by the parametric pump. The orange curve in Fig. 3 shows even flatter gain of $8.5 \pm 0.3 \mathrm{~dB}$ obtained in the same $25 \mathrm{~m}$ long fibre at pump power of $8 \mathrm{~W}$. In this case, the pump polarization was controlled to obtain the flattest gain possible. Using pump polarization to optimize the gain spectrum shape is a novel technique and will be discussed further at conference [12]. These two gain spectra are extremely flat and broad, but possess relatively low gain magnitude, even while using high pump power. We therefore aim to study 
techniques for increasing the FOPA gain magnitude, while keeping GV low and preferably with improved energy efficiency.

The simplest way to increase the gain magnitude is to increase the HNLF length as demonstrated by the purple curve in Fig. 3. This corresponds to the gain spectrum obtained using a $100 \mathrm{~m}$ long HNLF with pump power of $5 \mathrm{~W}$. The pump power is decreased compared to the previous two cases due to SBS threshold decrease with fibre length. A gain increase takes place at the cost of GV increase and BW decrease, providing a gain of $17.5 \pm 2 \mathrm{~dB}$ over $10.7 \mathrm{THz}$. Note that the Raman gain is substantially larger than the parametric gain in this case.

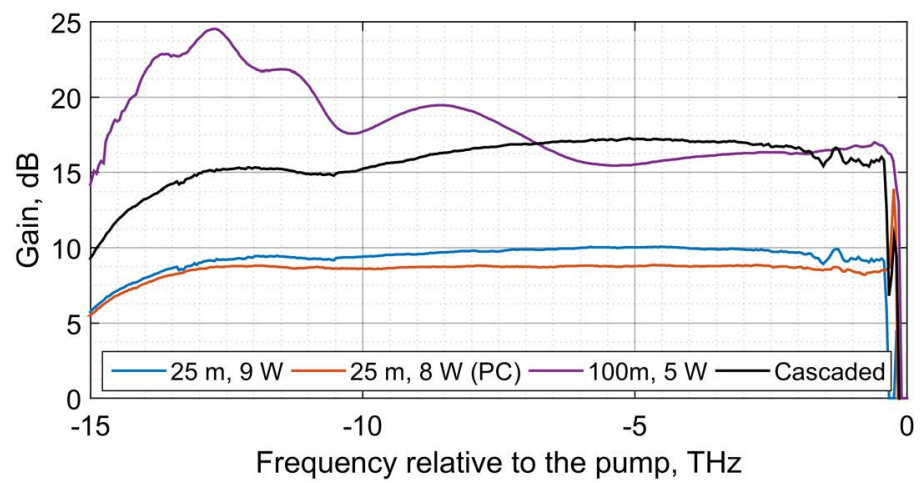

Figure 3. Experimental spectra for different HNLF length/pump power combinations: (a) $100 \mathrm{~m} / 5 \mathrm{~W}$ (purple trace), (b) 25m/9 W (blue trace), (c) $25 \mathrm{~m} / 8 \mathrm{~W}$ polarisation-tuned pump (red trace), (d) cascaded (black trace).

It can be seen that the GV increase in the $100 \mathrm{~m}$ fibre is much larger than the increase in gain magnitude. We therefore propose that the gain magnitude be increased by cascading multiple flat gain spectra. For example, two $25 \mathrm{~m}$ fibres could be placed sequentially with a filter between them to remove idlers of the first stage. This would lead to two independent FOPA stages, where the pump from the first stage can be reused in the second stage, thus improving energy efficiency. Assuming a filter loss of $1 \mathrm{~dB}$ and pump power in the first stage of $9 \mathrm{~W}$, the pump power in the second stage would therefore be $\sim 7 \mathrm{~W}$. The total gain in such a cascaded amplifier can be predicted by summing the gain spectra obtained in the $25 \mathrm{~m}$ fibre with pump powers of $9 \mathrm{~W}$ and $7 \mathrm{~W}$ and subtracting $1 \mathrm{~dB}$ to account for mid-stage loss. The resulting predicted cascaded gain spectrum is shown by the black curve in Fig. 3. Cascading suggests a gain of $16 \pm 1.2 \mathrm{~dB}$ over $13 \mathrm{THz}$, which is substantially flatter and broader than $100 \mathrm{~m}$ gain spectrum with similar gain magnitude. Therefore, the cascading of ultra-flat low gain spectra is viewed as a practical approach to reach high net gain over BW $>100 \mathrm{~nm}$ with low GV. It also should be noted that in case of quadratic gain and neglecting mid-stage loss, the cascading of two identical FOPA stages provides more gain than a single stage FOPA with the same total fibre length as long as nonlinear phase shift per stage $\gamma P L>\sqrt{2}$.

\section{RAMAN ENHANCEMENT OF FOPA}

Raman gain in the FOPA can arise from the parametric pump itself, or from an intentional extra pump. The use of the latter can provide extra gain, BW or improved signal performance [13]. However, the single-pump FOPA gain BW is typically much larger than that of a single-pump Raman. Therefore, multiple Raman pumps would be needed to provide impact over the whole parametric gain range. On the other hand, parametric pump generated Raman has to be considered whenever the parametric gain is wide enough to overlap with areas with notable Raman susceptibility. It has been shown that the amplitude of parametric gain starts to be notably affected by both imaginary and real parts of Raman susceptibility at offset of just over $5 \mathrm{THz}$ from the parametric pump [14]. Obviously, significant Raman gain will arise in the usual range (10-15 THz away from the pump in silica), where there is a high imaginary part of Raman susceptibility. It should be noted that Raman gain coefficient is typically 3-4 times smaller than phase-matched parametric gain coefficient [15], therefore Raman gain induced by the parametric pump often invokes localized peak on the Stokes side and dip on the anti-Stokes side, which increase GV across gain spectrum, but does not add much useable gain. Consequently, one reasonable way of exploiting the parametric pump induced Raman gain is to supress parametric gain peak or to fill a parametric gain dip. However, this has never been experimentally demonstrated. Another way is to extend parametric gain rolling off at $\sim 10 \mathrm{THz}$ away from the pump by Raman gain as was demonstrated in Fig. 3 . However, this requires a similar amplitude of Raman and parametric gain. Given the difference between Raman and FOPA gain coefficients, we found that it is possible to keep Raman and quadratic parametric gain on the same level up to $\sim 10 \mathrm{~dB}$ in a fibre with a measured Raman fractional strength $f_{R}=0.29$. Figure $4 \mathrm{a}$ demonstrates how Raman gain with various $f_{R}$ compares with phase-matched (blue curve) and quadratic (black curve) parametric gain plotted versus nonlinear phase shift $\gamma P L$. Raman gain was calculated as $G_{R}=\exp \left(2 \cdot f_{R} \gamma P L\right)$, quadratic gain as $G_{q}=(\gamma P L)^{2}+1$ and phase-matched gain as $G_{m}=[\cosh (\gamma P L)]^{2}$. It can be seen that the gain magnitude where quadratic parametric gain is equal to Raman gain (black dots) depends on $f_{R}$. Figure 3 demonstrates higher Raman gain than the one calculated and shown in Fig. 4, because in practice there is an overlapping and 
a complex interaction between parametric and Raman gains [14]. However, it is important that gain value where parametric and Raman gains are equal increases with decrease of $f_{R}$ (Fig. $4 \mathrm{~b}$ ). Therefore, it is expected that in fibres with low $f_{R}$ it is possible to match magnitudes of the Raman gain and the quadratic parametric gain up to $\sim 15-20 \mathrm{~dB}$.
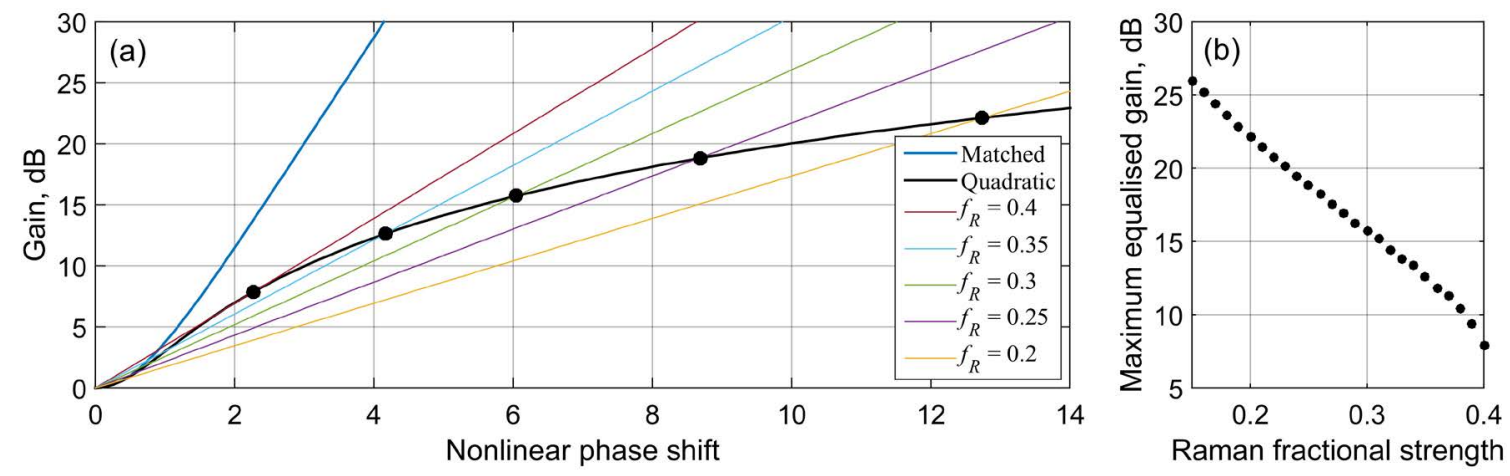

Figure 4. Analytical comparison of Raman and parametric gain:

(a) Raman gain with varied fractional strength and parametric gain (phase-matched and quadratic) are shown as a function of nonlinear phase shift. Black dots show where parametric and Raman gains are equal;

(b) Equal parametric and Raman gains as a function of the Raman fractional strength.

\section{CONCLUSIONS}

We have shown that cascading of two independent FOPA stages with broad and flat gain spectrum suggests lower GV comparing to single stage. We demonstrated that cascaded FOPA may have gain BW up to $13 \mathrm{THz}$ $(110 \mathrm{~nm})$, gain magnitude over $15 \mathrm{~dB}$ and $\mathrm{GV}$ of $2.4 \mathrm{~dB}$. Gain BW over $10 \mathrm{THz}$ in the suggested approach is attributed to Raman gain induced by the parametric pump.

\section{ACKNOWLEDGEMENTS}

The authors wish to thank Dr Ian McClean of II-VI for useful discussions and II-VI for CASE support of V. Gordienko. The work was funded by EPSRC project UPON, EP/M005283/1.

\section{REFERENCES}

[1] A.D. Ellis et al:: $4 \mathrm{~Tb} / \mathrm{s}$ transmission reach enhancement using $10 \times 400 \mathrm{~Gb} / \mathrm{s}$ super-channels and polarization insensitive dual band optical phase conjugation, IEEE J. Lightw. Technol., vol. 34, pp. 1717-1723, Jan. 2016.

[2] Y. Emori, K. Tanaka, and S. Namiki: $100 \mathrm{~nm}$ bandwidth flat-gain Raman amplifiers pumped and gain-equalised by 12wavelength-channel WDM laser diode unit, Electron. Lett., vol. 35, pp. 1355-1356, Aug. 1999.

[3] M. Jamshidifar, A. Vedadi, and M.E. Marhic: Continuous-wave one-pump fiber optical parametric amplifier with 270 nm gain bandwidth, in Proc. ECOC 2009, Vienna, Austria, Sep. 2009, paper 1.1.4.

[4] R. Malik et al.: Demonstration of ultra-wideband phase-sensitive fiber optical parametric amplifier, IEEE Photon. Technol. Lett., vol. 28, pp. 175-177, Jan. 2016.

[5] M.F.C. Stephens et al.: The impact of pump phase-modulation and filtering on WDM signals in a fibre optical parametric amplifier, in Proc. OFC 2015, Los Angeles, USA, Mar. 2015, paper W2A.43.

[6] M.F.C. Stephens, V. Gordienko, and N.J. Doran: $20 \mathrm{~dB}$ net-gain polarization-insensitive fiber optical parametric amplifier with >2 THz bandwidth, Opt. Express, vol. 25, pp. 10597-10609, May 2017.

[7] M.E. Marhic: Fiber optical Parametric Amplifiers, Oscillators and Related Devices, Cambridge University Press, 2008, Chap. 3.

[8] Y. Taniguchi et al.: Nonlinear optical fiber, nonlinear optical device, and optical signal processor, European Patent 1988411A1, 2008.

[9] V. Gordienko et al.: Novel broadband gain-spectrum measurement technique for Raman and parametric amplifiers, in Proc. OFC 2017, Los Angeles, USA, Mar. 2017, paper W2A.11.

[10] A. E. El-Taher et al.: High efficiency supercontinuum generation using ultra-long Raman fiber cavities, Opt. Express, vol. 17, pp. 17909-17915, 2009.

[11] V. Gordienko et al:: Ultra-flat wideband single-pump Raman-enhanced parametric amplification, Opt. Express, vol. 25, pp. 4810-4818, Mar. 2017.

[12] V. Gordienko et al.: Gain spectrum shaping technique for one-pump fibre optical parametric amplifier (FOPA), submitted to ECOC 2017, Gothenburg, Sweden, Sep. 2017.

[13] M.F.C. Stephens et al.: Improved WDM performance of a fibre optical parametric amplifier using Raman-assisted pumping, Opt. Express, vol. 23, pp. 902-911, Jan. 2015.

[14] A.S.Y. Hsieh et al:: Combined effect of Raman and parametric gain on single-pump parametric amplifiers, Opt. Express, vol. 15, pp. 8104-8114, June 2007.

[15] J. Hansryd et al.: Fiber-based optical parametric amplifiers and their applications, IEEE J. Sel. Topics. Quantum Electron., vol. 8, pp. 506-520, May/June 2002. 